\title{
FLOTROP - a biogeographical database on rangeland vegetation for northern tropical Africa
}

\author{
Philippe Daget
}

\begin{abstract}
FLOTROP (GIVD ID AF-00-005) is a vegetation-plot database that focusses on rangeland vegetation from the northern part of tropical Africa. Many studies on vegetation and vegetation dynamics of those rangelands have been conducted over the last six decades. However, the corresponding data are widely dispersed among scientists and institutions. FLOTROP aims therefore at compiling a joint database of historical and current vegetation plots from the region, in order to standardize the data formats and species nomenclature and to facilitate overarching ecological analyses.
\end{abstract}

Keywords: historical data set; pastoral vegetation; plot database; synoptic table; tropical Africa; vegetation map.

GIVD Database ID: AF-00-005

Last update: $2012-05-10$

\section{FLOTROP}

Scope: The scope of FLOTROP is to be able to answer the questions "Who saw what, where and when", to preserve old phytosociological observations and to present interpretations of the relevés preserved, all this in the pastoral vegetation of Northern Tropical Africa.

Status: emerging

Period: 1929-2007

Database manager(s): Philippe Daget (philippe.daget@gmail.com, philippe.lecomte@cirad.fr)

Owner: CIRAD-SELMET

Web address: http://umr-selmet.cirad.fr/les-produits-et-expertises/produits/flotrop

Availability: free online Online upload: [NA]

Database format(s): DYALOG APL

Export format(s): [NA]

Publication: GASTON, A. \& Ph. DAGET, 1999, Flotrop : Constitution d'une base de données sur les pâturages d'Afrique tropicale septentrionale, Sécheresse 10: 183-189.

Plot type(s): normal plots Plot-size range: [NA]

Non-overlapping plots: 34,533

Estimate of existing plots: [NA]

Completeness: [NA]

Total plot observations: 34,533

Number of sources: 256

Valid taxa: 5,852

Countries: BF: 21.5\%; BJ: 3.1\%; CF: 0.7\%; Cl: 5.4\%; CM: 4.0\%; CV: 1.5\%; DJ: 1.2\%; EG: 0.8\%; ET: $2.0 \%$; GH: $3.0 \%$; GN: 0.9\%; GW: $<0.1 \%$; LR: 0.9\%; LY: 0.8\%; MA: 0.8\%; ML: 5.8\%; MR: 7.5\%; NE: 10.6\%; NG: 1.9\%; SD: $1.8 \%$; SL: 0.9\%; SN: 15.8\%; SO: $2.0 \%$; TD: 12.4\%; TG: 3.0\%

Forest: [NA] - Non-forest: [NA]

Guilds: all vascular plants: $100 \%$

Environmental data: altitude: $60 \%$

Performance measure(s): presence/absence only: $60 \%$; cover: $8 \%$; other: $32 \%$

Geographic localisation: point coordinates less precise than GPS, up to $1 \mathrm{~km}: 100 \%$

Sampling periods: 1920-1929: 0.2\%; 1930-1939: 2.2\%; 1940-1949: 1.4\%; 1950-1959: 6.5\%; 1960-1969: 11.3\%; 1970-1979: 22.7\%; 1980-1989: 18.5\%; 1990-1999: 14.2\%; 2000-2009: $23.1 \%$

Information as of 2012-07-12; further details and future updates available from http://www.givd.info/ID/AF-00-005

Philippe Daget (philippe.daget@gmail.com, philippe.lecomte@cirad.fr)

CIRAD, TA 18/A - UR Elevage, 34398 Montpellier, FRANCE 